\title{
A semi-analytical solution for the mean wind profile in the Atmospheric Boundary Layer: the convective case
}

\author{
L. Buligon ${ }^{1}$, G. A. Degrazia ${ }^{1}$, O. C. Acevedo ${ }^{1}$, C. R. P. Szinvelski ${ }^{3}$, and A. G. O. Goulart ${ }^{2}$ \\ ${ }^{1}$ Universidade Federal de Santa Maria, Departamento de Física, Santa Maria, RS, Brazil \\ ${ }^{2}$ Universidade Federal do Pampa/UFSM, Centro de Ciências Exatas e Tecnológicas, Bagé, RS, Brazil \\ ${ }^{3}$ Centro de Educação Superior do Alto Vale do Itajaí, UDESC/CEAVI, Ibirama, SC, Brazil
}

Received: 26 June 2009 - Published in Atmos. Chem. Phys. Discuss.: 23 September 2009

Revised: 28 December 2009 - Accepted: 31 January 2010 - Published: 3 March 2010

\begin{abstract}
A novel methodology to derive the average wind profile from the Navier-Stokes equations is presented. The development employs the Generalized Integral Transform Technique (GITT), which combines series expansions with Integral Transforms. The new approach provides a solution described in terms of the quantities that control the wind vector with height. Parameters, such as divergence and vorticity, whose magnitudes represent sinoptic patterns are contained in the semi-analytical solution. The results of this new method applied to the convective boundary layer are shown to agree with wind data measured in Wangara experiment.
\end{abstract}

\section{Introduction}

The Navier-Stokes equations provide the framework for the interpretation of atmospheric boundary layer flows. However, their analytical solution requires approximations, which are, in many cases, idealized and distant from the physical reality. Particularly, the mean wind profile is a solution of the governing equations whose derivation can be applied to a wide variety of natural processes.

The classical Ekman expression for the mean wind profile is, probably, the most famous example of an analytical solution of the simplified Navier-Stokes equations (Sorbjan, 1989; Stull, 1988). Such solution needs, however, the strong, non-realistic assumption that the vertical eddy diffusivities $(K)$ are constant with height. In fact, Grisogono (1995) argues that “...it is a complicated, nonlinear function of the

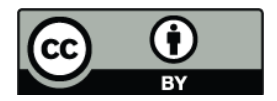

Correspondence to: G. A. Degrazia (degrazia@ccne.ufsm.br) flow structure and there is no explicit relation between the boundary-layer profiles and K".

The search for an analytical solution for the mean wind profiles in the atmospheric boundary layer under more realistic conditions has been a major focus of mathematical and physical research for a long time. For a given, imposed eddy diffusivity profile, solutions have been derived in a wide set of studies, reviewed by Zilitinkevich (1970), Monin and Yaglom (1971) and Grisogono (1995).

Other studies have expanded the solution to include baroclinic and advective conditions (Miles, 1994; Bannon and Salem, 1995). Berger and Grisogono (1998) extended the results obtained by Grisogono (1995) for the baroclinic case and a generic vertical eddy diffusivity profile. Tan (2001) proposed a semi-geostrophic Ekman layer solution for variable eddy diffusivities and baroclinicity. This model united the solutions presented by Wu and Blumen (1982) and Grisogono (1995), finding that the mean wind structure depends on the inertial acceleration, eddy diffusivity and baroclinic pressure gradient. The study concluded that anti-cyclonic wind shear accelerates the flow, while cyclonic shear has the opposite effect. Wilson and Flesch (2004) used a three-layer simplified model that provided a good comparison to observed wind profiles.

In the present study, using the Generalized Integral Transform Technique (GITT), we derive a semi-analytical solution of the Navier-Stokes equation to obtain the mean wind profile in the atmospheric boundary layer. Such technique is a hybrid numerical-analytical method applied to the treatment and solution of partial differential equations (Özişik, 1993; Mikhailov and Özişik, 1984; Cotta, 1993). It provides a systematic, direct and efficient approximation to the solution of homogeneous and non-homogeneous, stationary and

Published by Copernicus Publications on behalf of the European Geosciences Union. 
non-stationary, linear and non-linear boundary-value problems. The technique combines series expansion and an integration employing an inverse-transform pair. The PBL is discretized into $N$ sub-intervals in such manner that inside each sub-region the eddy diffusivity is the average value (Moreira et al., 1999), that allows the use of realistic eddy diffusivity profiles, which depend on the physical characteristics of the energy-containing eddies. The nonlinear terms are written in terms of kinematical properties of the flow, such as divergence and vorticity, allowing the solutions to be interpreted in terms of large-scale synoptic conditions. The model results are compared to observed wind profiles obtained from the classical Wangara experiment (Clarke et al., 1971).

\section{Semi-analytical model}

\subsection{Basic equations}

Considering that the turbulent momentum fluxes can be parameterized by a first order closure (K-theory), the mean horizontal wind spatial distribution is given by the NavierStokes equation in the following form:

$$
\begin{aligned}
u \frac{\partial u}{\partial x}+v \frac{\partial u}{\partial y} & =f_{c} v-f_{c} v_{g}+\frac{\partial}{\partial x}\left(K_{x} \frac{\partial u}{\partial x}\right) \\
& +\frac{\partial}{\partial y}\left(K_{y} \frac{\partial u}{\partial y}\right)+\frac{\partial}{\partial z}\left(K_{z} \frac{\partial u}{\partial z}\right), \\
u \frac{\partial v}{\partial x}+v \frac{\partial v}{\partial y} & =f_{c} u_{g}-f_{c} u+\frac{\partial}{\partial x}\left(K_{x} \frac{\partial v}{\partial x}\right) \\
& +\frac{\partial}{\partial y}\left(K_{y} \frac{\partial v}{\partial y}\right)+\frac{\partial}{\partial z}\left(K_{z} \frac{\partial v}{\partial z}\right),
\end{aligned}
$$

with $u \equiv u(x, y, z), v \equiv v(x, y, z), z_{0}<z<z_{i}, 0<x<L_{x}$ and $0<y<L_{y}$. The $f_{c}$ is the Coriolis parameter.

The equations above assume stationarity, no mean vertical motion, and that the molecular dissipation terms are neglectable. On the other hand, the flow is allowed to vary horizontally.

The geostrophic wind components in the baroclinic case are approximated by:

$u_{g}=u_{T} z+u_{g 0}$,

$v_{g}=v_{T} z+v_{g 0}$,

where $u_{g 0}$ and $v_{g 0}$ are the surface geostrophic winds components and $u_{T}$ and $v_{T}$ are the thermal wind components (Sorbjan, 1989).

The eddy diffusivities in each of the directions are represented by $K_{x}, K_{y}$ and $K_{z}$.

To realistically reproduce the wind profile, it is important to consider the vertical variation of the eddy diffusivities. As a consequence, in the present approach, the planetary boundary layer (PBL) is discretized into $N$ sublayers (Vilhena and Barichello, 1991; Moreira et al., 1999; Degrazia et al., 2001).
In each of the sublayers, the eddy diffusivities and horizontal wind components assume vertically averaged values. To overcome the difficulties that arise from the nonlinear character of Eqs. (1a) and (1b), the advective terms are written in terms of the large-scale kinematical properties of the flow (Bluestein, 1992), namely, divergence $(\delta)$ and vorticity $(\zeta)$ :

$$
\begin{aligned}
u_{n} \frac{\partial u_{n}}{\partial x}+v_{n} \frac{\partial u_{n}}{\partial y} & =\frac{\delta}{2} u_{n}-\frac{\zeta}{2} v_{n}, \\
u_{n} \frac{\partial v_{n}}{\partial x}+v_{n} \frac{\partial v_{n}}{\partial y} & =\frac{\delta}{2} v_{n}+\frac{\zeta}{2} u_{n} .
\end{aligned}
$$

Where the deformation components of the wind field were neglected and the index $n$ refers to the different sublayers considered. With the assumptions above, Eqs. (1a) and (1b) can be written as:

$$
\begin{aligned}
\frac{\delta}{2} u_{n}-\frac{\zeta}{2} v_{n} & =f_{c} v_{n}-f_{c} v_{g n}+K_{x n} \frac{\partial^{2} u_{n}}{\partial x^{2}}+K_{y n} \frac{\partial^{2} u_{n}}{\partial y^{2}} \\
& +K_{z n} \frac{\partial^{2} u_{n}}{\partial z^{2}}, \\
\frac{\delta}{2} v_{n}+\frac{\zeta}{2} u_{n} & =f_{c} u_{g n}-f_{c} u_{n}+K_{x n} \frac{\partial^{2} v_{n}}{\partial x^{2}}+K_{y n} \frac{\partial^{2} v_{n}}{\partial y^{2}} \\
& +K_{z n} \frac{\partial^{2} v_{n}}{\partial z^{2}},
\end{aligned}
$$

with $z_{n} \leq z \leq z_{n+1}, \quad 0<x<L_{x}, \quad 0<y<L_{y}$ and $n=$ $1,2, \ldots, N$.

Multiplying Eq. (4b) by $i(i \in \mathbb{C})$, and adding term by term to Eq. (4a), yields

$$
\begin{aligned}
K_{x n} \frac{\partial^{2} W_{n}}{\partial x^{2}}+K_{y n} \frac{\partial^{2} W_{n}}{\partial y^{2}}+K_{z n} \frac{\partial^{2} W_{n}}{\partial z^{2}} \\
-\left[\frac{\delta}{2}+\left(f_{c}+\frac{\zeta}{2}\right) i\right] w_{n}=-f_{c} W_{g n} i,
\end{aligned}
$$

where $w_{n}=u_{n}+v_{n} i, w_{g n}=u_{g n}+v_{g n} i, z_{n} \leq z \leq z_{n+1}, 0<$ $x<L_{x}, 0<y<L_{y}$ and $n=1,2, \ldots, N$.

Equation (5) is a differential equation on the complex variable function $w_{n}=u_{n}+v_{n} i$, whose solution provides an expression to the mean wind profile in terms of the flow divergence and vorticity.

\subsection{Boundary and interface conditions}

The horizontal wind speeds are assumed to be constant at the lower boundary at $z=z_{0}$, and to be geostrophic at the upper boundary, the PBL top. Laterally, a horizontal domain is assumed, with dimensions $L_{x} \times L_{y}$. At the lateral boundaries, the wind components are given by the imposed divergence and vorticity, so that:

$$
\begin{aligned}
& w_{n}=w_{0} \quad \text { in } \quad z=z_{0} \quad \text { and } \quad n=1, \\
& w_{n}=w_{g n} \quad \text { in } \quad z=z_{i} \quad \text { and } \quad n=N,
\end{aligned}
$$


and

$W_{n}=\frac{1}{2}(-\zeta+\delta i) y \quad$ in $\quad x=0$,

$w_{n}=\frac{1}{2}(\delta+\zeta i) L_{x}+\frac{1}{2}(-\zeta+\delta i) y \quad$ in $\quad x=L_{x}$

$W_{n}=\frac{1}{2}(\delta+\zeta i) x, \quad$ in $\quad y=0$,

$W_{n}=\frac{1}{2}(\delta+\zeta i) x+\frac{1}{2}(-\zeta+\delta i) L_{y} \quad$ in $\quad y=L_{y}$.

At the interfaces between neighbor vertical layers, it is necessary to assume continuity of both the eddy fluxes and horizontal wind components.

$\begin{aligned} W_{n} & =W_{n+1}, \\ K_{z n} \frac{\partial W_{n}}{\partial z} & =K_{z(n+1)} \frac{\partial w_{n+1}}{\partial z},\end{aligned}$

with $z=z_{n}$ and $n=1,2, \ldots(N-1)$.

\section{Solution}

Equation (5) can be solved using the Generalized Integral Transform Technique - GITT (Mikhailov and Özişik, 1984; Özişik, 1993; Cotta, 1993). In this method, the solution function is expanded in terms of the eigenfunctions corresponding to the auxiliary problem (Sturm-Liouville), associated with the original problem. The eigenfunction orthogonality condition is used to determine the expansion coefficients, hence originating the integral transform and its inverse. Applying the integral transform, the partial derivatives in relation to variables $x$ and $y$ are removed, reducing the problem to an ordinary second-order differential equation on variable $z$. Therefore, once the transformed problem is solved, the inverse formula is used to obtain a solution to the original problem. The truncation order is selected according to the desired precision.

\subsection{The auxiliary problem}

The auxiliary problem associated with $w_{n}$ is:

$$
\begin{array}{r}
\frac{K_{x n}}{K_{z n}} \frac{\partial^{2} \psi\left(\lambda_{p q}, x, y\right)}{\partial x^{2}}+\frac{K_{y n}}{K_{z n}} \frac{\partial^{2} \psi\left(\lambda_{p q}, x, y\right)}{\partial y^{2}}= \\
-\lambda_{p q}^{2} \psi\left(\lambda_{p q}, x, y\right),
\end{array}
$$

with boundary conditions:

$\psi=0$ in $x=0$ and $x=L_{x}$,

$\psi=0$ in $y=0$ and $y=L_{y}$.

The solution to the problem above is (Mikhailov and Özişik, 1984; Özişik, 1993):

$$
\begin{aligned}
& \psi_{1}\left(\beta_{p}, x\right)=\sin \left(\beta_{p}^{\prime} x\right) \text { for } \quad p=1,2, \ldots \\
& \psi_{2}\left(\gamma_{q}, y\right)=\sin \left(\gamma_{q}^{\prime} y\right) \text { for } \quad q=1,2, \ldots
\end{aligned}
$$

$\beta_{p}=\frac{p \pi}{L_{x}} \sqrt{\frac{K_{x n}}{K_{z n}}}$ for $p=1,2, \ldots$

$\gamma_{q}=\frac{q \pi}{L_{y}} \sqrt{\frac{K_{y n}}{K_{z n}}}$ for $q=1,2, \ldots$

where $\beta_{p}^{\prime}=\sqrt{\frac{K_{z n}}{K_{x n}}} \beta_{p}$ and $\gamma_{q}^{\prime}=\sqrt{\frac{K_{z n}}{K_{y n}}} \gamma_{q}$ are the positive roots of equations $\sin \left(\beta_{p}^{\prime} L_{x}\right)=0$ and $\sin \left(\gamma_{q}^{\prime} L_{y}\right)=0$, respectively. Equations (11) and (12) are known, respectively, as the eigenfunctions and eigenvalues associated with the Sturm-Liouville problem. From the normalization integral:

$N\left(\lambda_{p q}\right)=\int_{0}^{L_{x}} \int_{0}^{L_{y}}\left[\psi\left(\lambda_{p q}, x^{\prime}, y^{\prime}\right)\right]^{2} d y^{\prime} d x^{\prime}$,

with $\quad \psi\left(\lambda_{p q}, x^{\prime}, y^{\prime}\right)=\psi_{1}\left(\beta_{p}, x^{\prime}\right) \psi_{2}\left(\gamma_{q}, y^{\prime}\right) \quad$ and $\lambda_{p q}^{2}=\beta_{p}^{2}+\gamma_{q}^{2}$. The corresponding norms can be obtained:

$\frac{1}{N\left(\beta_{p}\right)^{1 / 2}}=\sqrt{\frac{2}{L_{x}}}$ for $p=1,2, \ldots$

$\frac{1}{N\left(\gamma_{q}\right)^{1 / 2}}=\sqrt{\frac{2}{L_{y}}}$ for $q=1,2, \ldots$

\subsection{The transform problem}

The integral transform is given by:

$$
\begin{aligned}
\overline{W_{n}}\left(\lambda_{p q}, z\right)= & \frac{1}{N\left(\lambda_{p q}\right)^{1 / 2}} \int_{0}^{L_{x}} \int_{0}^{L_{y}} \\
& {\left[\psi\left(\lambda_{p q}, x^{\prime}, y^{\prime}\right) W_{n}\left(x^{\prime}, y^{\prime}, z\right)\right] d y^{\prime} d x^{\prime}, }
\end{aligned}
$$

with $N\left(\lambda_{p q}\right)=N\left(\beta_{p}\right) N\left(\gamma_{q}\right)$.

Applying the operator

$\frac{1}{N\left(\lambda_{p q}\right)^{1 / 2}} \int_{0}^{L_{x}} \int_{0}^{L_{y}} \psi\left(\lambda_{p q}, x^{\prime}, y^{\prime}\right) d y^{\prime} d x^{\prime}$ to Eq. (5) yields:

$$
\begin{aligned}
& \frac{1}{N\left(\lambda_{p q}\right)^{1 / 2}} \int_{0}^{L_{x}} \int_{0}^{L_{y}} \psi\left(\lambda_{p q}, x^{\prime}, y^{\prime}\right) \frac{\partial^{2} W_{n}}{\partial z^{2}} d y^{\prime} d x^{\prime} \\
& +\frac{1}{N\left(\lambda_{p q}\right)^{1 / 2}} \int_{0}^{L_{x}} \int_{0}^{L_{y}} \psi\left(\lambda_{p q}, x^{\prime}, y^{\prime}\right)
\end{aligned}
$$

$\left[\frac{K_{x n}}{K_{z n}} \frac{\partial^{2} W_{n}}{\partial x^{\prime 2}}+\frac{K_{y n}}{K_{z n}} \frac{\partial^{2} W_{n}}{\partial y^{\prime 2}}\right] d y^{\prime} d x^{\prime}$

$-\left[\frac{\delta}{2 K_{z n}}+\left(\frac{f_{c}}{K_{z n}}+\frac{\zeta}{2 K_{z n}}\right) i\right] \frac{1}{N\left(\lambda_{p q}\right)^{1 / 2}}$

$\int_{0}^{L_{x}} \int_{0}^{L_{y}} \psi\left(\lambda_{p q}, x^{\prime}, y^{\prime}\right) w_{n} d y^{\prime} d x^{\prime}=$

$-\frac{f_{c} W_{g n} i}{K_{z n}} \frac{1}{N\left(\lambda_{p q}\right)^{1 / 2}} \int_{0}^{L_{x}} \int_{0}^{L_{y}} \psi\left(\lambda_{p q}, x^{\prime}, y^{\prime}\right) d y^{\prime} d x^{\prime}$, 
with $w_{n} \equiv w_{n}\left(x^{\prime}, y^{\prime}, z\right)$ and $\lambda_{p q}^{2}=\beta_{p}^{2}+\gamma_{q}^{2}$.

Each term in Eq. (16) is solved by a different method. In the first integral, Lebniz rule is used. The second integral is solved by using Green's theorem, employing the eigenvalue problem (9), along with the boundary conditions (7). The third integral is directly substituted by definition (15). Finally, in the right-hand side, results (11) and (12) are used. This procedure yields in the following ordinary differential equation:

$\frac{d^{2} \overline{W_{n}}}{d z^{2}}-\left(\alpha_{1 n}+\alpha_{2 n} i\right) \overline{W_{n}}=-\frac{f_{c} W_{g n} G i}{K_{z n}}-C_{n}$,

where the constants are given in Appendix A.

The boundary and interface conditions associated with the transformed problem in $z$ direction can be determined applying definition (15) to equations (6) and (8) given, respectively, by:

$\overline{W_{n}}=G W_{0} \quad$ in $\quad z=z_{0} \quad$ and $\quad n=1$,

$\overline{W_{n}}=G W_{g n} \quad$ in $\quad z=z_{i} \quad$ and $\quad n=N$,

$$
\begin{gathered}
\overline{W_{n}}=\overline{w_{n+1}}, \\
K_{z n} \frac{\partial \overline{W_{n}}}{\partial z}=K_{z(n+1)} \frac{\partial \overline{W_{n+1}}}{\partial z}, \\
\text { with } z=z_{n} \text { and } n=1,2, \ldots(N-1) .
\end{gathered}
$$

\subsection{The inverse transform}

From the GITT formalism, the solution to equation (5) is given by the expansion:

$w_{n}(x, y, z)=\sum_{p=1}^{\infty} \sum_{q=1}^{\infty} \frac{\psi\left(\lambda_{p q}, x, y\right)}{N\left(\lambda_{p q}\right)^{1 / 2}} \overline{W_{n}}\left(\lambda_{p q}, z\right)$.

The solution to Eq. (17) is given by:

$$
\begin{aligned}
\overline{W_{n}}\left(\lambda_{p q}, z\right)=A_{n} \exp \left[r_{1 n} z\right]+B_{n} \exp \left[r_{2 n} z\right] & \\
& +\overline{W_{n}} P\left(\lambda_{p q}, z\right),
\end{aligned}
$$

where $A_{n}, B_{n}, r_{1 n}, r_{2 n}, \overline{W_{n}} p \in \mathbb{C}$. The constants are given in Appendix A.

The boundary and interface conditions lead to the determination of constants $A_{n}$ and $B_{n}$. Therefore, for each $p$ and $q$, the resulting system is solved numerically.

Using the previous results in the Eq. (20), results

$$
\begin{array}{r}
w_{n}(x, y, z)=\sum_{p=1}^{\infty} \sum_{q=1}^{\infty} \frac{2}{\sqrt{L_{x} L_{y}}} \sin \left(\beta_{p}^{\prime} x\right) \sin \left(\gamma_{q}^{\prime} y\right) \\
\overline{W_{n}}\left(\lambda_{p q}, z\right),
\end{array}
$$

where $\overline{W_{n}}\left(\lambda_{p q}, z\right)$ is given by the Eq. (21).
Finally, we obtain the components of the average wind, $u_{n}$ and $v_{n}$, from the fact that $w_{n}(x, y, z)=u_{n}(x, y, z)+$ $v_{n}(x, y, z) i$. In that case,

$u_{n}(x, y, z)=\Re W_{n}(x, y, z)$,

$v_{n}(x, y, z)=\Im w_{n}(x, y, z)$,

where $\Re$ represents the real part of $W_{n}$, and $\Im_{n}$ represents the imaginary part of $w_{n}$.

\section{Eddy diffusivity}

The eddy diffusivity vertical profiles employed in this study have been proposed by Degrazia et al. (2000). Such eddy diffusivities are based on Taylor's statistical diffusion theory, in which the shear buoyancy PBL spectra are modeled by means of a linear combination of the convective and mechanical forcings. Therefore, in the present case, such parameterization allowed reproducing the realistic case of a convective boundary layer where shear-generated turbulence occurs. The eddy diffusivities for such conditions are given by the following expression:

$K_{\alpha}=0.11 \sqrt{c_{i}}\left[\frac{z \psi_{\epsilon}^{1 / 3} w_{*}\left(z / z_{i}\right)^{1 / 3}}{\left(f_{m}^{*}\right)_{i}^{4 / 3}}+\frac{u_{*} z \phi_{\epsilon}^{1 / 3}}{\left(f_{m}\right)_{i}^{4 / 3}}\right]$,

where $c_{i}=\alpha_{i} \alpha_{u}(2 \pi \kappa)^{-2 / 3}$ with $\alpha_{u}=0.5 \pm 0.05$ (Champagne et al., 1977; Sorbjan, 1989) and $\alpha_{i}=1, \frac{4}{3}, \frac{4}{3}$ for $u, v$ and $w$ components, respectively. $\quad w_{*}=\left(u_{*}\right)_{0}\left(\frac{z_{i}}{\kappa|L|}\right)^{1 / 3}$ is the convective velocity scale; $u_{*}=\left(u_{*}\right)_{0}^{2}\left(1-\frac{z}{h}\right)^{\alpha_{1}}$ is the local friction velocity, in which $\alpha_{1}=1.7$ (Wyngaard et al., 1974); $\left(u_{*}\right)_{0}$ is the superficial friction velocity; $z_{i}$ is the convective PBL height; $z$ is the height above the surface; $\psi_{\epsilon}=\frac{\epsilon_{b} z_{i}}{w_{*}^{3}}$ is the adimensional dissipation rate functions, $\epsilon_{b}=(0.75)^{3 / 2}\left(w_{*}^{3} / z_{i}\right)$ is the buoyant rate of TKE dissipation; $\phi_{\epsilon}=\frac{\epsilon_{s} k z}{u_{*}^{3}}$ is the adimensional molecular dissipation rate functions associated with mechanical productions, $\epsilon_{s}=\frac{u_{*}^{3}}{k z}\left(1-\frac{z}{z_{i}}\right)$ is the mechanical rate of TKE dissipation (Højstrup, 1982); $L$ is the Monin-Obukov length and $\kappa=0.4$ is the von Kármán constant; $\left(f_{m}^{*}\right)_{i}=\frac{z}{\left(\lambda_{m}\right)_{i}}$ is the reduced frequency of the convective spectral peak, where $\left(\lambda_{m}\right)_{i}$ is the peak wavelength of the turbulent velocity spectra. According to Kaimal et al. (1976) and Degrazia and Anfonssi (1998), $\left(\lambda_{m}\right)_{u}=\left(\lambda_{m}\right)_{v}=1.5 z_{i}$ and $\left(\lambda_{m}\right)_{w}=1.8 z_{i}\left[1-\exp \left(\frac{-4 z}{z_{i}}\right)-0.0003 \exp \left(\frac{8 z}{z_{i}}\right)\right]$.

The reduced frequency of the neutral peak, $\left(f_{m}\right)_{i}$, with $\left(f_{m}\right)_{u}=0.045\left(1+117 \frac{f_{c} z}{\left(u_{*}\right)_{0}}\right)$, 
Table 1. Meteorological Parameters for the experiment of Wangara.

\begin{tabular}{ccccccc}
\hline Day & Hour & $-L(\mathrm{~m})$ & $z_{i}(\mathrm{~m})$ & $u_{*}(\mathrm{~m} / \mathrm{s})$ & $u_{g 0}(\mathrm{~m} / \mathrm{s})$ & $v_{g 0}(\mathrm{~m} / \mathrm{s})$ \\
\hline 33 & $15: 00$ & 2.8 & 1200 & 0.155 & -5.32 & -0.77 \\
40 & $15: 00$ & 10 & 1200 & 0.14 & 1.7 & -2.55
\end{tabular}

$\left(f_{m}\right)_{v}=0.16\left(1+33 \frac{f_{c} z}{\left(u_{*}\right)_{0}}\right)$ and

$\left(f_{m}\right)_{w}=0.35\left(1+15 \frac{f_{c} z}{\left(u_{*}\right)_{0}}\right) ; f_{c}=2 \Omega \sin \phi$ is the Coriolis parameter.

\section{Result}

\subsection{Analysis parameters}

Equation (22) expresses, in terms of many independent parameters, the mean wind profiles. Among these parameters are: the size of the horizontal area defined by $L_{x}$ and $L_{y}$, the thickness $\Delta z$ of the vertical sublayers over which the PBL was divided, the truncation order ( $p$ and $q$ ), and the values of large-scale divergence $\delta$ and vorticity $\zeta$, which affect the lateral boundary conditions.

The size of the horizontal domain has an appreciable impact on the solution, but only for small areas (Fig. 1). As $L_{x}$ and $L_{y}$ are successively increased from $1 \mathrm{~km}$ to $100 \mathrm{~km}$, the solution becomes independent of the domain size for $L_{x}=L_{y} \geq 50 \mathrm{~km}$. It means that the present solution is meaningful only over horizontal areas as large as $50 \mathrm{~km}$. From this point on, the solutions shown were obtained with $L_{x}=L_{y}=50 \mathrm{~km}$.

A similar convergence analysis was applied to the sublayer thickness $\Delta z$ and the truncation orders $p$ and $q$, leading to the conclusion that $\Delta z=5 \mathrm{~m}$ and $p=q=9$ are values that warrant convergence of the mean wind profiles. The model results depend on the horizontal position within the domain, even when no large-scale divergence and vorticity are considered (Fig. 2). The horizontal variation is larger close to the domain boundaries, so that there is a good portion of the domain, near its center, for which the wind profiles do not vary largely in the horizontal. The following analysis considers the vertical profiles at the domain center only.

\subsection{Comparison to observational data from the Wangara experiment}

The Wangara experiment was conducted in Hay, New South Wales, Australia, from July to August, 1967 (Clarke et al., 1971). Wind profiles were obtained every hour up to a $2-\mathrm{km}$ height, using pilot balloons. A 16-m tower provided micrometeorological surface observations. In the present study, two convective days were chosen for comparison to the proposed model: days 33 and 40 (Table 1).
The wind components at the top of the domain are given by a thermal wind approximation (Eq. 2), and both the surface geostrophic winds $\left(u_{g 0}\right.$ and $\left.v_{g 0}\right)$ and the thermal wind magnitudes $\left(u_{T}\right.$ and $\left.v_{T}\right)$ are given by Wangara observed values.

The mean wind magnitudes simulated by the model are similar to the average magnitudes observed at Wangara (Fig. 3). It is important to stress that such agreement concerns only the vertical overall average, but not the local maxima and minima observed at day 33 , which characterize an unmixed wind profile. Indeed, such vertical variability is quite difficult to capture with a simplified model, as stated by Wyngaard (1988): “unfortunately, our knowledge of PBL physics does not yet allow us to calculate the wind profile from first principles ...". Unmixed wind profiles, such as those observed at day 33, may be attributed to a number of reasons, such as local baroclinicity or vertical eddy diffusivity variability. Any of these reasons are, however, casespecific, and cannot be reproduced by a model where thermal wind is assumed to be constant.

The simulated values for different large-scale synoptic conditions (in terms of divergence and vorticity) cover a range of wind magnitudes, generally in agreement with the observations. Far from the surface, the condition without divergence and vorticity is the one which departs mostly from the measurements. This same condition, on the other hand, provides the best match to observations at the lowest levels (inlet). Different combinations of vorticity and divergence have been applied for the comparison. There is no clear distinction among most of them, as can be seen in Fig. 3. However, an analysis based on statistical indices (Appendix B) reveals that, while the results are very similar for the wind magnitude (Table 2), the approximation for wind direction (Table 3 ) is improved when both the divergence and vorticity are positive.

Regarding the vertical profiles for day 33 (Fig. 3), the analysis based on statistical indices shows that, when $\delta=\zeta=0$ and $\delta=\zeta=-f_{c}$, the model overestimates the mean observed wind magnitude (small negative values of $F B$ ). On the other hand, the statistical index $F B$ shows that the horizontal wind direction is underestimated regardless of $\delta$ and $\zeta$, meaning that the modeled winds are rotated counterclockwise with respect to the observations. The statistical index $F S$ indicates that, except for the case $\delta=\zeta=0$, the dispersion of the mean wind magnitude underestimated the experimental data. For the wind direction, this same index is negative in all cases, a consequence of the very small wind 

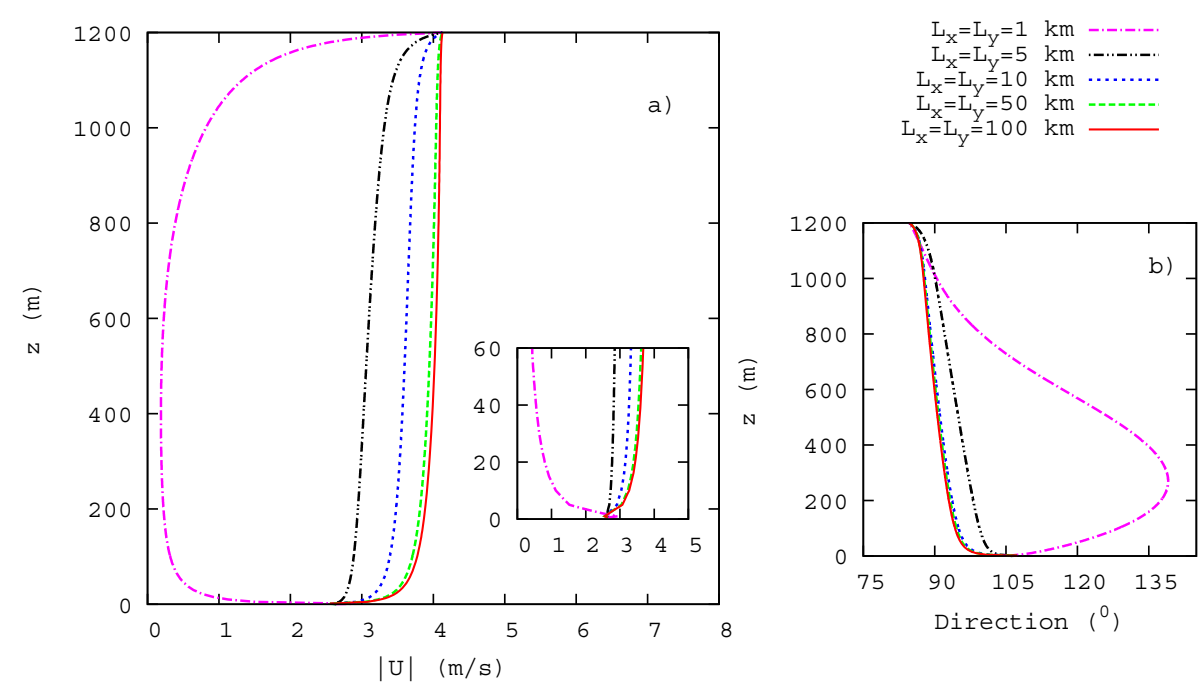

Fig. 1. Simulated vertical profiles of (a) wind magnitude and (b) wind direction, for different domain sizes, as indicated in legend. Inlet shows the wind magnitude vertical profile for the lowest $60 \mathrm{~m}$ in detail.
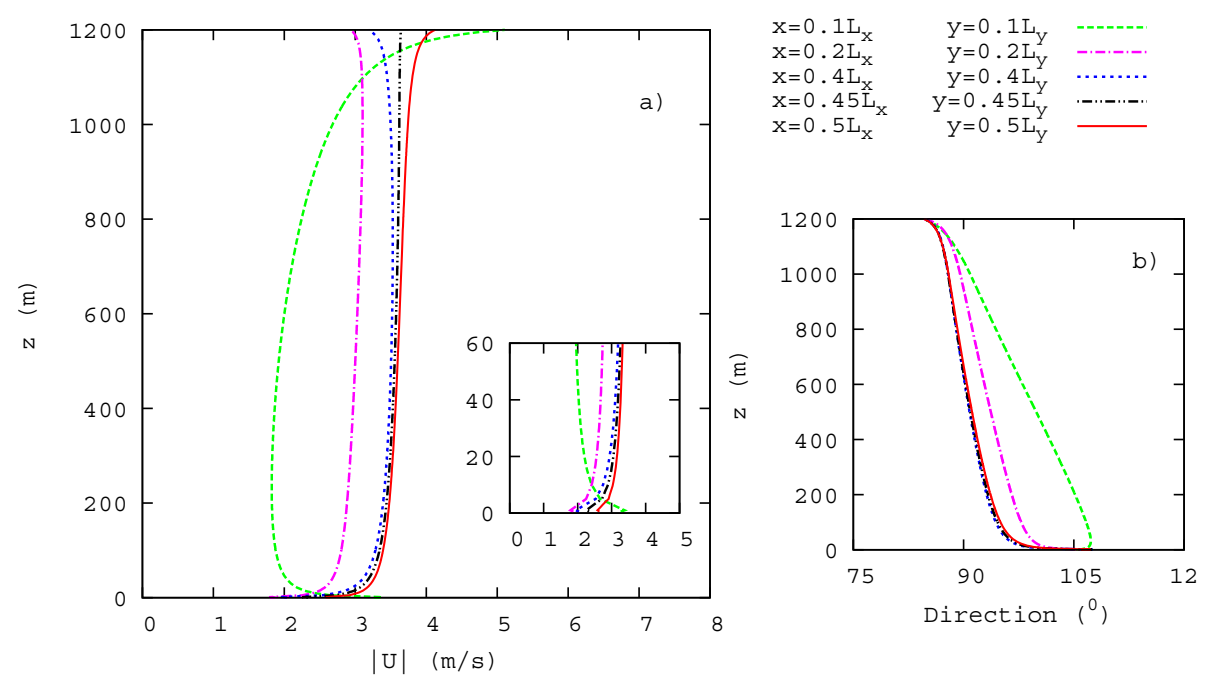

Fig. 2. Simulated vertical profiles of (a) wind magnitude and (b) wind direction, for different positions within the domain, as indicated in legend. Inlet shows the wind magnitude vertical profile for the lowest $60 \mathrm{~m}$ in detail.

direction variability with height in the observed data, while the model results indicate a slight wind rotation with height. Other indices, such as NMSE, and FA2 are similar for all cases, and indicative of good agreement between model and observations. Finally, the correlation coefficient $R$ was more variable, and therefore, serves as a measure of the best agreement in each case.

Thermal winds were observed only twice a day, at synoptic times, and those values were interpolated to 15:00 LT . The large gradients near the top of the boundary layer arise from the assumed baroclinicity. For any case, the different modeled profiles agree to each other as a consequence of the top boundary conditions. They do not necessarily agree to the observed winds at the boundary layer top as a consequence of the interpolation used to calculate the thermal wind. This limitation has been noticed by Sorbjan (1989): "Finally, results of the Wangara experiment pointed out the difficulties and limitations of obtaining accurate measurements of thermal winds, vertical velocities, and representative spatially averaged fluxes".

Similar results were obtained for day 40 (Fig. 4). Indeed, the statistical indices (Tables 4 and 5) indicate that the model reproduced the observations better than for day 33 . In this case, the condition without divergence and vorticity showed the largest departure from the observations for wind magnitude both at upper and lower levels. Again, the best 

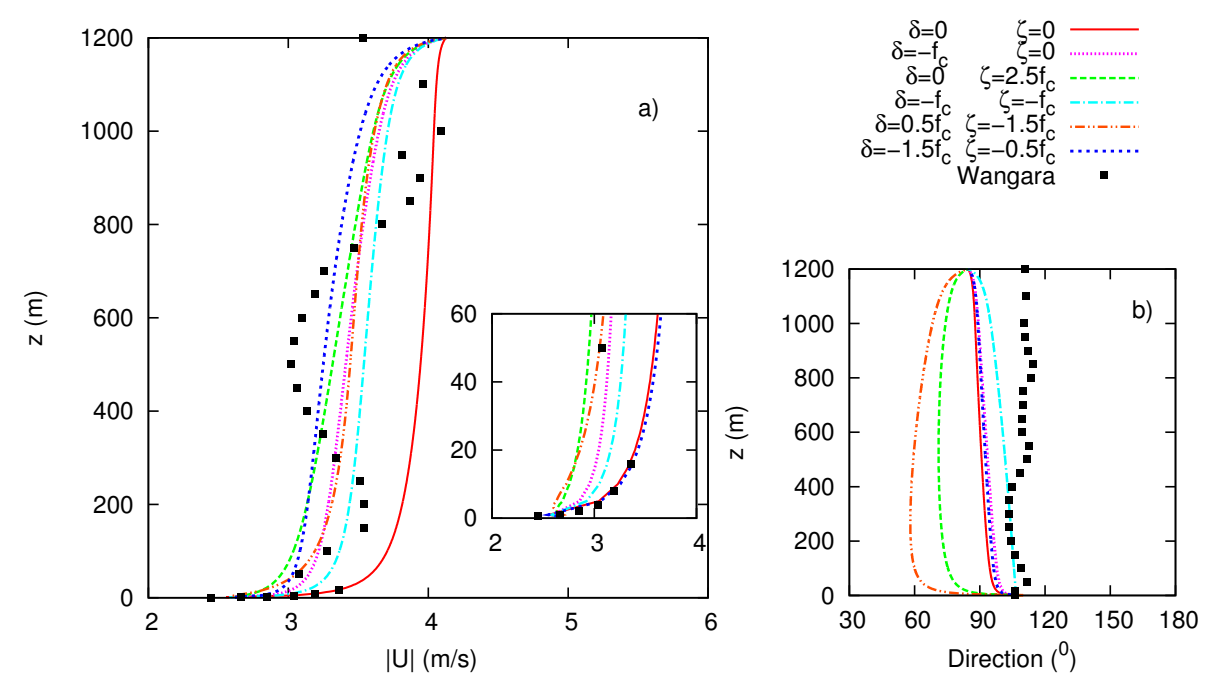

Fig. 3. Simulated vertical profiles of (a) wind magnitude and (b) wind direction for Wangara day 33, and different values of wind divergence and vorticity, as indicated in legend. Inlet shows the wind magnitude vertical profile for the lowest $60 \mathrm{~m}$ in detail. Squares represent data from the Wangara experiment for day 33. At Wangara, $f_{c}=-8.2 \times 10^{-5} \mathrm{~s}^{-1}$.

Table 2. Statistical indices evaluating the wind magnitude profiles shown in Fig. 3.

\begin{tabular}{lccccc}
\hline Wind Speed $(m / s)$ & NMSE & $F B$ & $F S$ & $R$ & $F A 2$ \\
\hline$\delta=\zeta=0$ & 0.021 & -0.107 & -0.183 & 0.685 & 1.000 \\
$\delta=-f_{c} ; \zeta=0$ & 0.007 & 0.004 & 0.110 & 0.719 & 1.000 \\
$\delta=0 ; \zeta=2.5 f_{c}$ & 0.009 & 0.032 & 0.067 & 0.688 & 1.000 \\
$\delta=-f_{c} ; \zeta=-f_{c}$ & 0.008 & -0.024 & 0.071 & 0.711 & 1.000 \\
$\delta=0.5 f_{c} ; \zeta=-1.5 f_{c}$ & 0.009 & 0.010 & 0.039 & 0.650 & 1.000 \\
$\delta=-1.5 f_{c} ; \zeta=-0.5 f_{c}$ & 0.009 & 0.040 & 0.193 & 0.702 & 1.000 \\
\hline
\end{tabular}

Table 3. Statistical indices evaluating the wind direction profiles shown in Fig. 3.

\begin{tabular}{lccccc}
\hline Direction $\left({ }^{0}\right)$ & NMSE & $F B$ & $F S$ & $R$ & $F A 2$ \\
\hline$\delta=\zeta=0$ & 0.029 & 0.151 & -0.583 & -0.526 & 1.000 \\
$\delta=-f_{c} ; \zeta=0$ & 0.023 & 0.129 & -0.535 & -0.579 & 1.000 \\
$\delta=0 ; \zeta=2.5 f_{c}$ & 0.123 & 0.318 & -1.121 & -0.309 & 1.000 \\
$\delta=-f_{c} ; \zeta=-f_{c}$ & 0.010 & 0.068 & -0.446 & -0.599 & 1.000 \\
$\delta=0.5 f_{c} ; \zeta=-1.5 f_{c}$ & 0.235 & 0.437 & -1.301 & -0.172 & 1.000 \\
$\delta=-1.5 f_{c} ; \zeta=-0.5 f_{c}$ & 0.014 & 0.090 & -0.470 & -0.619 & 1.000 \\
\hline
\end{tabular}

representation offered by the model for both wind magnitude and direction occurred with positive values of divergence and vorticity. Furthermore, the solutions with negative vorticity provided the worst approximation for wind direction. A similar analysis of the statistical indices as that made for day 33 can be made for day 40 (Tables 4 and 5).
In both days 33 and 40, the best approximations to the observed profiles were obtained for positive vorticity and divergence (notice that the Coriolis parameter is negative), consistent with the occurrence of anti-cyclonic large-scale flow at the period. Indeed, the synoptic surface pressure charts (Clarke et al., 1971) indicate the presence of a high-pressure system at the region, for both days 33 and 40. Such consistency is further evidence that the model is able to reproduce the wind profile realistically. 

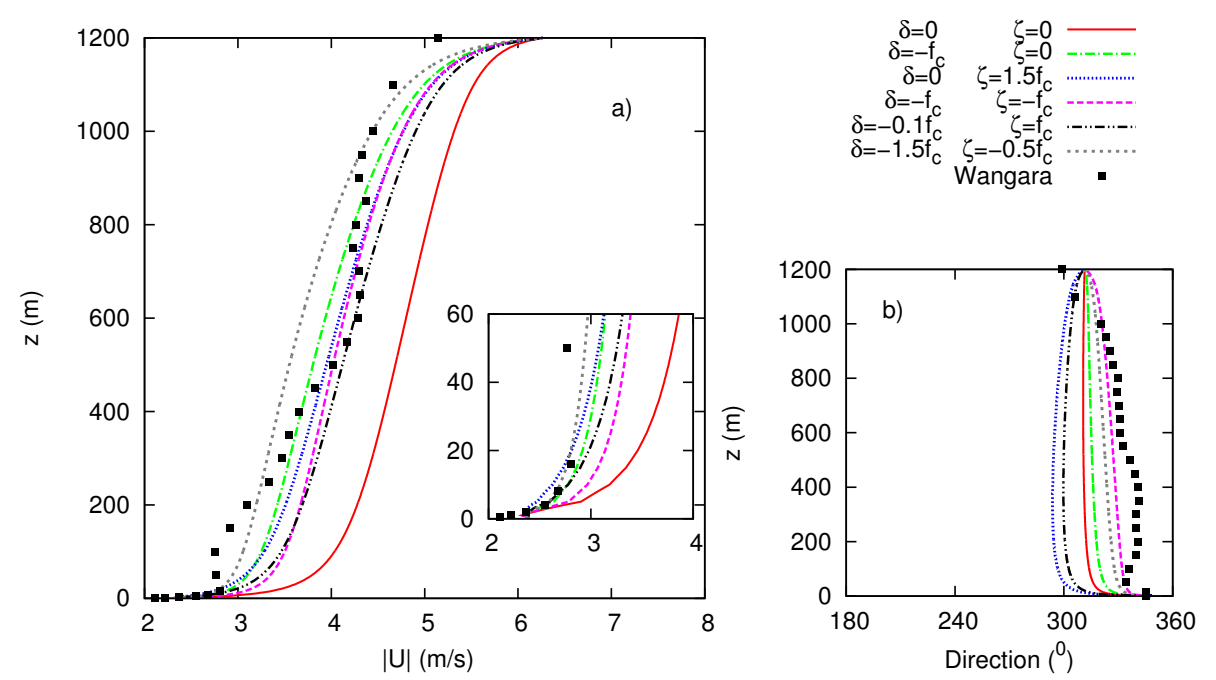

Fig. 4. Simulated vertical profiles of (a) wind magnitude and (b) wind direction for Wangara day 40, and different values of wind divergence and vorticity, as indicated in legend. Inlet shows the wind magnitude vertical profile for the lowest $60 \mathrm{~m}$ in detail. Squares represent data from the Wangara experiment for day 40. At Wangara, $f_{c}=-8.2 \times 10^{-5} \mathrm{~s}^{-1}$.

Table 4. Statistical indices evaluating the wind magnitude profiles shown in Fig. 4.

\begin{tabular}{lccccc}
\hline Wind Speed $(m / s)$ & NMSE & $F B$ & $F S$ & $R$ & $F A 2$ \\
\hline$\delta=\zeta=0$ & 0.042 & -0.187 & -0.179 & 0.952 & 1.000 \\
$\delta=-f_{c} ; \zeta=0$ & 0.007 & -0.028 & -0.038 & 0.941 & 1.000 \\
$\delta=0 ; \zeta=1,5 f_{c}$ & 0.008 & -0.047 & -0.095 & 0.954 & 1.000 \\
$\delta=-f_{c} ; \zeta=-f_{c}$ & 0.011 & -0.071 & -0.038 & 0.946 & 1.000 \\
$\delta=-0.1 f_{c} ; \zeta=f_{c}$ & 0.012 & -0.083 & -0.107 & 0.960 & 1.000 \\
$\delta=-1.5 f_{c} ; \zeta=-0.5 f_{c}$ & 0.010 & 0.022 & -0.001 & 0.914 & 1.000 \\
\hline
\end{tabular}

Table 5. Statistical indices evaluating the wind direction profiles shown in Fig. 4.

\begin{tabular}{lccccc}
\hline Direction $\left({ }^{0}\right)$ & NMSE & $F B$ & $F S$ & $R$ & $F A 2$ \\
\hline$\delta=\zeta=0$ & 0.004 & 0.052 & 0.005 & 0.493 & 1.000 \\
$\delta=-f_{c} ; \zeta=0$ & 0.002 & 0.040 & 0.101 & 0.604 & 1.000 \\
$\delta=0 ; \zeta=1.5 f_{c}$ & 0.011 & 0.091 & -0.364 & 0.288 & 1.000 \\
$\delta=-f_{c} ; \zeta=-f_{c}$ & 0.001 & 0.013 & 0.340 & 0.850 & 1.000 \\
$\delta=-0.1 f_{c} ; \zeta=f_{c}$ & 0.008 & 0.078 & -0.249 & 0.346 & 1.000 \\
$\delta=-1.5 f_{c} ; \zeta=-0.5 f_{c}$ & 0.001 & 0.023 & 0.244 & 0.763 & 1.000 \\
\hline
\end{tabular}

\section{Conclusions}

In the present study, a novel approach was used to obtain the average wind profile from the Navier-Stokes equations. The method is based on the Generalized Integral Transform Technique (GITT), applied to the convective boundary layer discretized in sublayers. Such discretization allows using eddy diffusivities that vary vertically. GITT is a procedure that combines series development and integral transforms, leading to a final solution (Eq. 22) that contains the physical parameters determining the wind variability with height.
Large-scale kinematical flow properties, such as divergence and vorticity are included in the solution, through the boundary conditions and the nonlinear advective terms of the original equations.

The model provided a good comparison to the observed data from Wangara experiment. The mean wind magnitudes are similar, although the model is not able to reproduce the unmixed character of the profiles. This is, however, a very complex task, not achieved by previous analytical results that provide a very good approximation, such as those by Wilson and Flesch (2004). 
The results may be improved further employing more realistic boundary conditions. Besides, the selection of other eddy diffusivity profiles may lead to improved solutions. In this sense, the methodology developed in the present study is generic, allowing other eddy diffusivity profiles to be considered.

The main aim of this study is to establish an alternative method to determine the mean wind profiles. The method has been shown in detail, as well as its validation in comparison to observed data. From this point, it can be used for a further examination of a more generalized problem. As an example, in the approach taken here no temporal evolution is considered. The non-stationary problem can be solved using GITT along with Laplace transform applied to the time. Besides, the examples here were restricted to the convective case, but the development allows the use of the same approach for any stability condition. The use of appropriate eddy diffusivity profiles may lead to the determination of wind profiles under stable conditions as well.

\section{Appendix A}

\section{Constants}

$$
\begin{aligned}
& \alpha_{1 n}=\lambda_{p q}^{2}+\frac{\delta}{2 K_{z n}}, \\
& \alpha_{2 n}=\frac{f_{c}}{K_{z n}}+\frac{\zeta}{2 K_{z n}}, \\
& G=\frac{2}{\sqrt{L_{x} L_{y}}}\left[\left(\frac{1-\cos (p \pi)}{\beta_{p}^{\prime}}\right)\left(\frac{1-\cos (q \pi)}{\gamma_{q}^{\prime}}\right)\right],
\end{aligned}
$$

$$
C_{n}=C_{3}+C_{6} \text {, }
$$

$$
\begin{aligned}
C_{3} & =\frac{2}{\sqrt{L_{x} L_{y}}} \frac{K_{x n}}{K_{z n}}\left[C_{1} L_{y} \frac{\cos (q \pi)}{\gamma_{q}^{\prime}}\right. \\
& \left.-C_{2}\left(\frac{1-\cos (q \pi)}{\gamma_{q}^{\prime}}\right)\right],
\end{aligned}
$$

$$
C_{1}=-\frac{1}{2}(-\zeta+\delta i) \beta_{p}^{\prime}[1-\cos (p \pi)],
$$

$$
C_{2}=\frac{1}{2}(\delta+\zeta i) L_{x} \beta_{p}^{\prime} \cos (p \pi)
$$

$$
C_{6}=\frac{2}{\sqrt{L_{x} L_{y}}} \frac{K_{y n}}{K_{z n}}\left[C_{4} L_{x} \frac{\cos (p \pi)}{\beta_{p}^{\prime}}\right.
$$

$$
\left.-C_{5}\left(\frac{1-\cos (p \pi)}{\beta_{p}^{\prime}}\right)\right] \text {, }
$$

$C_{4}=-\frac{1}{2}(\delta+\zeta i) \gamma_{q}^{\prime}[1-\cos (q \pi)]$,

$C_{5}=\frac{1}{2}(-\zeta+\delta i) L_{y} \gamma_{q}^{\prime} \cos (q \pi)$

$$
\begin{aligned}
& r_{1 n}=\left(\alpha_{1 n}^{2}+\alpha_{2 n}^{2}\right)^{1 / 4}\left[\cos \left(\frac{\theta_{n}}{2}\right)+i \sin \left(\frac{\theta_{n}}{2}\right)\right], \\
& r_{2 n}=\left(\alpha_{1 n}^{2}+\alpha_{2 n}^{2}\right)^{1 / 4}\left[\cos \left(\frac{\theta_{n}}{2}+\pi\right)+i \sin \left(\frac{\theta_{n}}{2}+\pi\right)\right], \quad(\mathrm{A} 5) \\
& \alpha_{1 n} \neq 0 \text { and } \alpha_{2 n} \neq 0, \theta_{n}=\arctan \left(\frac{\alpha_{2 n}}{\alpha_{1 n}}\right) ; \theta_{n} \neq \frac{\pi}{2}+\kappa \pi, \kappa=0,1 \\
& \overline{W_{n} P}\left(\lambda_{p q}, z\right)=\frac{1}{\left(\alpha_{1 n}^{2}+\alpha_{2 n}^{2}\right)}\left[\left(\alpha_{2 n}+\alpha_{1 n} i\right) \frac{f_{c} W_{g n} G}{K_{z n}}\right. \\
& \left.+\left(\alpha_{1 n}-\alpha_{2 n} i\right) C_{n}\right]
\end{aligned}
$$

\section{Appendix B}

\section{Statistical indices}

Following Hanna (1989) the statistical indices used in this study are defined as:

$$
\begin{aligned}
& \text { NMSE }=\frac{\overline{\left(C_{o}-C_{p}\right)^{2}}}{\overline{C_{o}} \overline{C_{p}}} \quad \text { (Normalized Mean Square Error) } \\
& F B=\frac{\left(\overline{C_{o}}-\overline{C_{p}}\right)}{0.5 \overline{\left(\overline{C_{o}}+\overline{C_{p}}\right)} \quad \text { (Fractional Bias) }} \\
& F S=2 \frac{\left(\sigma_{o}-\sigma_{p}\right)}{\left(\sigma_{o}+\sigma_{p}\right)} \quad(\text { Standard Fractional Bias) } \\
& R=\frac{\left(C_{o}-\overline{C_{o}}\right)\left(C_{p}-\overline{C_{p}}\right)}{\left(\sigma_{o} \sigma_{p}\right)} \quad \text { (Correlation Coefficient) } \\
& F A 2=0.5 \leq \frac{C_{o}}{C_{p}} \leq 2 \quad \text { (Factor of 2) }
\end{aligned}
$$

where $C$ is the analyzed amount and the subscript $o$ and $p$ refer to observed and predicted quantities, respectively, the over bar indicates an averaged value. The statistical index $F B$ says if the predicted quantity underestimates or overestimates the average observed ones. The statistical index NMSE represents the quadratic error of the predicted quantities related to the observed ones. The statistical index $F S$ indicates the as the model gets to simulate the dispersion of the observed data. The statistical index $F A 2$ supply the fraction of the data $(\%)$ for the ones which $0.5 \leq \frac{C_{o}}{C_{p}} \leq 2$. The best results are expected to have values near zero for the indices NMSE, $F B$ and $F S$ and near 1 in the indices $R$ and FA2. 
Acknowledgements. This work was partially supported by $\mathrm{CNPq}$ and CAPES.

Edited by: G. Vaughan

\section{References}

Bannon, P. R. and Salem, T. L. Jr.: Aspects of the baroclinic boundary layer, J. Atmos. Sci., 52, 574-596, 1995.

Berger, B. W. and Grisogono, B.: The Baroclinic, Variable Eddy Viscosity Ekman Layer, Bound.-Lay. Meteorol., 87, 363-380, 1998.

Bluestein, H. B.: Principles of Kinematics and Dynamics. Vol. I. Synoptic - Dynamic Meteorology in Midlatitudes, Oxford University Press, 608 pp., 1992.

Clarke, R. H., Dyer, A. J., Brook ,R. R., Reid, D. G. and Troup, A. J.: The Wangara Experiment: Boundary Layer Data, Division of Meteorological Physical Technical Paper - CSIRO- Austrália, 19, 1-339, 1971.

Champagne, F. H., Friehe, C. A., Larve, J. C., and Wyngaard, J. C.: Flux measurements, flux estimation techniques, and fine scale turbulence measurements in the instable surface layer over land, J. Atmos. Sci., 34, 515-520, 1977.

Cotta, R. M.: Integral transforms in computational heat and fluid flow, CRC Press, Boca Raton, FL, 340 pp., 1993.

Degrazia, G. A. and Anfonssi,D.: Estimation of the Kolmogorov constant $C_{0}$ from classical statistical diffusion theory, Atmos. Environ., 22, 3611-3614, 1998.

Degrazia, G. A., Anfonssi, D., Carvalho, J. C., Mangia, C., Tirabassi, T., and Campos Velho, H. F.: Turbulence parameterisation for PBL dispersion models in all stability conditions, Atmos. Environ., 34, 3575-3583, 2000.

Degrazia, G. A., Moreira, D. M. and Vilhena, M. T.: Derivation of an eddy diffusity depending on source distance for vertically inhomogeneous turbulence in a convective boundary layer, J. Appl. Meteor., 40, 1233-1240, 2001.

Grisogono, B. A: generalized Ekman layer profile with gradually varying eddy diffusivities, Q. J. Roy. Meteor. Soc., 121, 445453, 1995.

Hanna, S. R.: Confidence limits for air quality model evaluations as estimed by bootstrap and backife resampling methods, Atmos. Environ., 23, 1385-1398, 1989.
Højstrup, J.: Velocty spectra in the unstable surface planetary boundary layer, J. Atmos. Sci., 39, 2239-2248, 1982.

Kaimal, J. C., Wyngaard, D. A., Haugen, D. A., Cot, O. R., Izumi, Y., Caughey, S. J. and Readings, C. J.: Turbulence structure in the Convective Boundary Layer, J. Atmos. Sci., 33, 2152-2226, 1976.

Mikhailov, M. D. and Özişik, M. N.: Unified Analysis and Solutions of Heat and Mass Diffusion, John Wiley \& Sons Inc., New York, 524 pp., 1984.

Miles, J.: Analytical solutions for the Ekman layer, Bound.-Lay. Meteorol., 121, 1-10, 1994.

Monin, A. S. and Yaglom, A. M.: Statistical Fluid Mechanics, Mit Press, Cambridge, 769 pp., 1971.

Moreira, D. M., Degrazia, G. A. and Vilhena, M. T.: Dispersion from low sources in a convective boundary layer: an analytical model, Nuovo Cimento, 22C, 685-691, 1999.

Özişik, M. N.: Heat Conduction, John Wiley \& Sons Inc., New York, 692 pp., 1993.

Sorbjan, Z.: Structure of the atmospheric boundary layer, Prentice Hall, New Jersey, 317 pp., 1989.

Stull, R. B.: An Introduction to Boundary Layer Meteorology, Kluwer Academic Publishers, New Jersey, 666 pp., 1988.

Tan, Z.-M.: An Approximate Analytical Solution for the Baroclinic and Variable Eddy Diffusivity Semi-Geostrophic Ekman Boundary Layer, Bound.-Lay. Meteorol., 98, 361-385, 2001.

Venkatram, A., Wyngaard, J. C.: Lectures on Air Pollution Modeling, American Meteorological Society, Boston, 390 pp., 1988.

Vilhena, M. T. and Barichello, L. B.: A new analytical approach to solve the neutron transport equation, Kerntechnik, 56, 334-336, 1991.

Zilitinkevich, S. S.: Dynamics of Atmospheric Boundary Layer, Gidrometeoizdat, Leningrad, 192 pp., 1970.

Wilson, J. D. and Flesch, T. K.: An Idealized Mean Wind Profile for the Atmospheric Boundary Layer, Bound.-Lay. Meteorol., 110, 281-299, 2004.

Wu, R. and Blumen, W.: An analysis of Ekman boundary layer dynamics incorporating the geostrophic momentum approximation, J. Atmos. Sci., 39, 1774-1782, 1982.

Wyngaard, J. C., Cote, O. R., and Rao, K. S.: Modelling of the Atmospheric Boundary Layer.Advances, In Geo., 18A, 193-212, 1974.

Wyngaard, J. C.: Structure of the PBL, in: Lecture on Air Pollution Modeling, edited by: Venkatram, A. and Wyngaard, J. C.: American Meteorological Society, Boston, 390 pp., 1988. 